Service social

\title{
Famille, valeurs et société
}

\section{Claire Chamberland}

Volume 36, numéro 2-3, 1987

Approches intégrées

URI : https://id.erudit.org/iderudit/706363ar

DOI : https://doi.org/10.7202/706363ar

Aller au sommaire du numéro

\section{Éditeur(s)}

École de service social de l'Université Laval

\section{ISSN}

1708-1734 (numérique)

Découvrir la revue

\section{Citer cet article}

Chamberland, C. (1987). Famille, valeurs et société. Service social, 36(2-3), 274-285. https://doi.org/10.7202/706363ar

\section{Résumé de l'article}

De nombreuses études américaines et canadiennes associent pauvreté et mauvais traitements envers les enfants. En plus des aspects économiques de la pauvreté, il faut accorder une attention particulière aux carences des réseaux personnels et sociaux des familles : des environnements dépourvus de ressources et de services favorisent la violence familiale, surtout si les individus adhèrent à un système de valeurs néo-libérales et matérialistes. La réduction de la violence doit passer par une réorientation profonde des valeurs et des systèmes politiques et économiques, ainsi que par un apport accru de l'État aux parents. 
Chamberland, Claire, professeure à l'École de service social de l'Université de Montréal.

\section{Famille, valeurs et société}

\section{Claire Chamberland}

Parmi les problèmes d'adaptation observés dans les familles, la violence envers les enfants ainsi que l'absence ou la carence des soins qui leur sont apportés évoquent une réalité qui soulève bien des passions, fait couler de l'encre et provoque beaucoup d'épuisement. L'engorgement des services de protection de l'enfance n'a d'égal que l'impuissance des intervenant(e)s à remédier à une situation familiale détériorée et souvent désespérée. En 1983, aux États-Unis, on rapportait aux services concernés 21.6 enfants par 1000 (American Humane Association, 1985). À Montréal, durant cette même année, on a estimé que 12.04 signalements fondés, par 1000 enfants, ont été acheminés à la Direction de la protection de la jeunesse. ${ }^{1}$ On peut donc estimer que le problème est deux fois plus important chez les Américains que chez les Montréalais.

Dans une première perspective, on peut attribuer l'exaspération des parents (ou leur manque d'implication) envers leurs enfants à un manque de compétence personnelle. Le problème se limiterait ici à un agrégat d'individus perturbés. Le remède serait alors une intervention curative limitée au système familial ou encore le retrait systématique des enfants de leur famille... et son démantèlement à plus ou moins long terme. Dans une seconde perspective, la prévalence des mauvais traitements dans un milieu reflète en grande partie la qualité de l'environnement dans lequel ces problèmes émergent.

En démontrant l'impact des conditions d'appauvrissement économique et social des familles sur l'ampleur du phénomène des mauvais traitements envers les enfants, nous nous proposons de questionner du même coup les choix de société qui les produisent, tant dans la rationalisation des projets économiques que dans les systèmes de valeurs sur lesquels ils reposent, menaçant ainsi sérieusement le développement social et l'évolution des relations harmonieuses entre parents et enfants. 
À cet égard, l'approche écologique nous fournit un cadre théorique pertinent à une compréhension plus globale de la problématique, où les facteurs individuels et environnementaux interagissent d'une manière dynamique et souvent complexe. Pour saisir comment se structure l'environnement des individus, Bronfenbrenner (1979) propose en effet une classification originale en quatre systèmes sociaux interdépendants : les microsystèmes, qui constituent les milieux de vie fréquentés par les membres de la famille; le mésosystème, référant aux interrelations entre les microsystèmes; l'exosystème, qui se compose de contacts formels ou informels non fréquentés par la famille; et enfin, le macrosystème, englobant l'ensemble des valeurs, normes, codes d'éthique ou modèles de conduite entérinés par la collectivité. En somme, selon cette perspective, les comportements abusifs et négligents des parents sont largement tributaires des conditions physiques, sociales et économiques, ainsi que des contextes de vie (Belsky, 1980; Bouchard, 1981; Chamberland et Beaudry, sous presse). Nous nous intéresserons plus particulièrement ici à démontrer l'influence des déterminants exosystémiques (politiques sociales, pauvreté, stratégies économiques) et macrosystémiques (idéologie sociale) sur l'expérience de vie des familles et sur la prévalence des mauvais traitements.

\section{Pauvreté économique et mauvais traitements}

De très nombreuses études descriptives ont mis en évidence un lien étroit entre les conduites abusives et négligentes de parents et le contexte de pauvreté dans lequel ils se retrouvent (Garbarino et Crouter, 1978 ; Gil, 1970 ; Martin et Messier, 1981 ; Pelton, 1978 ; Strauss, Gelles et Steinmetz, 1980). D'après une étude américaine effectuée à l'échelle nationale, les enfants provenant de couches plus défavorisées sont plus susceptibles d'être maltraités : le taux d'incidence s'y élève à 27 enfants sur 1000 (Garbarino, 1984). À Montréal, pour l'année 1983, on a estimé que 16.8 enfants sur 1000 vivant dans des secteurs pauvres avaient été signalés à la Direction de la protection de la jeunesse pour cause de mauvais traitements, alors que la proportion passait à 5 enfants sur 1000 dans les secteurs plus favorisés (Bouchard, Chamberland et Beaudry, 1986).

D'après l'étude américaine de Garbarino et Crouter (1978), les facteurs économiques expliquent $62 \%$ de la variance observée dans les taux d'incidence enregistrés dans les différentes régions administratives 
d'une ville du Nebraska. À Montréal, pour des territoires de grandeur équivalente, nous avons observé une corrélation de 0.84 entre le pourcentage de familles en situation de pauvreté et le taux d'incidence mesuré à partir de l'identification des nouveaux cas durant les premiers mois de l'année 1983.

De plus, les caractéristiques généralement reliées à de plus grands risques de mauvais traitements sont également associées à la pauvreté, comme la présence de bébés de petit poids, prématurés ou difficiles à soigner, ou encore d'enfants handicapés (Egbuonu et Starfield, 1982; Nersesian et al., 1985). De même observe-t-on une proportion de mères présentant un profil d'apathie-futilité singulièrement plus élevé, tant chez les familles signalées que dans les couches de population les plus défavorisées (Polansky et al., 1979). Enfin, les familles monoparentales et celles où une femme est soutien financier sont également associées à des facteurs de risque. À l'instar de l'étude américaine de Garbarino et Crouter (1978), Chamberland, Bouchard et Beaudry (1986) ont mis en évidence une corrélation de 0.71 et de 0.75 respectivement entre, d'une part, les taux d'incidence observés dans différents territoires montréalais de centres locaux de services communautaires (C.L.S.C.) et, d'autre part, le pourcentage de familles monoparentales et de familles dont une femme est soutien financier de ces mêmes territoires. D'après les dernières études établissant le profil de pauvreté au Canada, ce risque de pauvreté est également plus élevé chez les familles dirigées par une femme seule : en 1983, une famille monoparentale sur deux, dont une femme est le seul soutien, vivait sous le seuil de pauvreté (Conseil national du Bien-être social, 1985).

Il est donc de plus en plus difficile, et pour le moins audacieux, de continuer à prétendre que le phénomène des mauvais traitements envers les enfants s'observe indifféremment chez les riches comme chez les pauvres.

\section{Pauvreté sociale et mauvais traitements}

La pauvreté économique ne représente toutefois pas le seul paramètre pour évaluer la qualité relative de l'environnement des familles. On ne peut en effet soutenir que celle-là entraîne automatiquement de l'abus ou de la négligence chez des familles économiquement démunies. La présence de ressources dans le voisinage ou dans les réseaux personnels peut atténuer l'impact de conditions de vie défavorables et ainsi faciliter 
l'harmonisation des rapports entre parents et enfants (Cochran et Brassard, 1979; Rohner et Nielsen, 1978). Ainsi Garbarino et Sherman (1980) ont effectivement constaté qu'à pauvreté égale, le voisinage où l'on retrouve le plus de cas de mauvais traitements présente certaines caractéristiques délétères : absence ou manque de fiabilité des systèmes de garde, isolement d'un plus grand nombre de familles, à l'écart des réseaux d'aide et exposées à une accumulation d'événements critiques.

Une étude microsociologique effectuée au printemps 1984 a permis d'étayer largement ce point de vue. Chamberland et al. (1986) ont, dans un premier temps, repéré deux voisinages ${ }^{2}$ défavorisés et présentant des caractéristiques macro-socio-économiques ${ }^{3}$ équivalentes, mais dont les taux d'incidence d'enfants maltraités contrastaient significativement. En 1983, les taux observés pour une période de six mois étaient de 17.98 familles sur 1000 pour le secteur à risque élevé, et de 2.49 familles sur 1000 pour le secteur à faible risque. Dans un deuxième temps, nous avons esquissé un portrait des ressources sociales disponibles dans le réseau personnel ou dans l'environnement des familles résidant dans ces deux voisinages. Pour ce faire, nous avons interviewé 80 mères du secteur à risque élevé et 73 de celui à faible risque et leur avons demandé quelle était leur perception de la qualité du soutien disponible dans leur environnement social. Les résultats sont sans équivoque. ${ }^{4}$ Les mères du voisinage à risque élevé disent pouvoir compter sur un moins grand nombre d'individus pour leur fournir du soutien affectif ou normatif, être plus souvent dans la situation de ne trouver personne pour leur fournir ce même soutien et être plus régulièrement sans aide alors qu'elles auraient besoin d'une assistance concrète. Elles rapportent, entre autres, subir plus fréquemment la présence de personnes nuisibles, lesquelles occupent, dans une plus large proportion, une place relativement centrale dans leur réseau (par exemple, les amies). Les personnes identifiées dans leur réseau répondent à une diversité plus restreinte de besoins. En effet, le réseau des mères du voisinage à risque élevé semble davantage constitué de relations plus ténues ou moins variées. Les membres de la parenté sont moins susceptibles d'apporter un soutien (affectif, instrumental ou normatif) alors que les professionnels sont plus souvent identifiés comme les aidants.

Les mères des familles résidant dans le voisinage à risque élevé estiment, de plus, qu'elles ne peuvent pas compter autant sur les ressources sociales de leur voisinage et elles les évaluent plus négativement. Elles considèrent leurs voisins moins chaleureux, sont moins satisfaites des contacts qu'elles entretiennent avec eux, considèrent qu'il est plus difficile de s'en faire des ami(e)s et, qu'en général, les gens se portent mutuellement moins d'intérêt. Elles estiment également que 
le milieu est globalement moins convenable pour élever leur enfant: elles sont moins satisfaites des endroits de jeu, des activités de loisirs, jugent les adultes moins tolérants envers les enfants et qu'il est plus difficile de recevoir de l'aide en tant que parents. Enfin, l'enfant aurait lui aussi accès à un environnement social moins adéquat : les mères du voisinage à risque élevé perçoivent qu'il est plus difficile pour l'enfant de s'y faire des amis et que l'influence des autres enfants sur le leur semble moins positive.

Bref, l'environnement social de ces mères, moins soutenant et plus conflictuel, exacerberait une situation déjà critique du point de vue des risques de mauvais traitements envers les enfants. Ce double appauvrissement, économique et social, engendrerait un niveau de tension intolérable pour les parents. Dans de telles conditions, les relations parent-enfant peuvent difficilement être imperméables au stress que subissent les parents, et dans ce cas, les enfants risquent de faire les frais de telles pressions.

Toutefois, même si l'habitabilité sociale de certains voisinages urbains joue un rôle important pour modérer l'impact de stresseurs socio-économiques, il n'en demeure pas moins que cet élément est extrêmement volatile, fragile et soumis aux caprices souvent imprévisibles des planificateurs du marché immobilier et de la finance. Des voisinages autrefois socialement sains sont aujourd'hui nos secteurs à risques plus élevés. Ainsi, certains facteurs économiques ont des impacts non négligeables sur le développement du tissu social en milieu urbain. Et qui plus est, dans le présent contexte économique, l'importance accordée à l'autosuffisance et à l'indépendance des individus peut même empêcher que se développent, entre les gens, des solidarités et une motivation à partager et à s'entraider.

En bref, on peut dire, avec une marge de certitude convenable, que la pauvreté économique et l'absence d'un tissu social soutenant constitueraient des éléments clés dans la configuration de facteurs généralement associés à des taux élevés d'abus et de négligence envers les enfants.

\section{Réalités canadienne et américaine}

Cette association pauvreté/mauvais traitements semble encore plus vraisemblable si l'on considère d'autres faits. Conformément à ce qui a déjà été mentionné, il y a approximativement deux fois plus de cas rapportés aux différents services de protection de l'enfance dans l'ensemble des États-Unis qu'à ceux de Montréal. Nous pouvons à tout le moins présumer que ce dernier taux est relativement représentatif des 
grands centres urbains canadiens. ${ }^{5}$ Malgré une paupérisation croissante chez les familles défavorisées canadiennes ${ }^{6}$ et américaines, ${ }^{7}$ et ce depuis 1979, les conditions économiques des familles pauvres semblent plus précaires et moins bien soutenues par l'État chez nos voisins américains. En effet, alors que $20 \%$ des familles considérées comme les plus pauvres (dernier quintile) se partageaient, en 1983,6.2\% du revenu national (Conseil national du Bien-être social, 1985), leurs analogues américaines se contentaient, en 1984, de $4.2 \%$ de l'ensemble du revenu national. Pour contrer l'effet néfaste de la récession économique sur la situation des familles canadiennes pauvres, les systèmes de sécurité sociale fédéral et provincial ont permis, en 1983, de hausser leur revenu de $58 \%$, par le biais de différents types de transferts gouvernementaux.

\section{Le néo-libéralisme économique : l'indépendance des pauvres... à tout prix}

Il en va toutefois autrement chez les Américains. L'État se désengage progressivement face à ses pauvres. Les coupures drastiques effectuées dans les secteurs de la sécurité sociale et en éducation, au profit de crédits plus importants accordés à la défense (Garfinkel et Haveman, 1986), ajoutées à des mesures fiscales favorisant les plus nantis et défavorisant les plus démunis, expliqueraient, en partie du moins, l'écart de plus en plus grand entre les riches et les pauvres. Cette situation reflète bien l'idéologie néo-libérale largement répandue chez les dirigeants et dans les milieux d'affaires américains et qui gagne malheureusement du terrain dans plusieurs pays occidentaux.

On admet bien, dans ces milieux, qu'il existe une surreprésentation de situations problématiques chez les populations les plus défavorisées : criminalité, chômage, mauvais traitements, familles monoparentales, taux élevé de mortalité infantile, etc. Mais loin de blâmer les conditions d'expansion économique qui génèrent l'inégalité et l'inéquité dans la redistribution des ressources, on attribue plutôt l'origine de ces problèmes à une transformation significative des valeurs morales chez certaines catégories de la population, en particulier chez les jeunes noirs pauvres (Murray, 1984). On soutient que les efforts consentis par les gouvernements depuis le milieu des années 1960 pour lutter contre la pauvreté ("The Great Society ") ont bouleversé le code d'éthique traditionnel des Américains, fondé sur le travail, la solidarité familiale et l'ascension sociale (la recherche de statut). Le soutien que l'État peut apporter aux plus démunis entraîne non seulement une homogénéisation dans la 
population (par opposition à une société composée de classes sociales distinctes), mais engendrerait un accroissement de comportements dépendants. Le blâme est non plus attribué aux conditions structurelles de la pauvreté, mais plutôt à la perte de dignité des pauvres et de volonté d'échapper à leur condition.

De l'avis des tenants du néo-libéralisme, une motivation fondée sur cette idéologie a permis aux États-Unis de devenir une grande puissance, et c'est cette même motivation qu'il faut une fois de plus réintroduire dans la population. On propose des mesures qui encouragent les jeunes à s'élever et devenir les meilleurs, afin de modifier le contexte "négatif » dans lequel certains d'entre eux se sont développés depuis la fin des années 1960. L'encouragement systématique de comportements autosuffisants et compétitifs est tributaire d'une idéologie sociale fondée sur des prémisses néo-darwiniennes. Les conditions de survie reposent surtout sur les volontés individuelles, les mécanismes de sélection étant soumis aux impératifs souvent sauvages des lois du marché.

\section{Violence, négligence et système économique}

Ces conditions que nous venons de mentionner sont intrinsèquement porteuses de violence car elles cautionnent, non pas le développement de l'initiative personnelle (ce qui serait certes souhaitable), mais le combat pour l'appropriation de ressources envers et contre tous et contribuent à la création d'environnements stressants et compétitifs. Comment ne pas être terrifiés par l'omniprésence de la violence physique et verbale dans les agglomérations urbaines où, pour survivre, les gens doivent quotidiennement s'imposer et s'affronter. Comment ne pas être bouleversés de voir, à Chicago, des gamins de moins de 10 ans se promener avec des armes à feu pour se protéger (Paré, 1986); de constater qu'entre $85 \%$ et $97 \%$ des parents disent avoir utilisé la punition physique à un moment ou l'autre de la vie de l'enfant (Bouchard, 1981); ou encore d'apprendre que $48 \%$ des assauts très violents envers des enfants sont perpétrés par les membres de la fratrie (Garbarino, 1983)? C'est ce qui faisait dire à Gil (1970) que la violence, dans nos familles, est le reflet de l'autoritarisme et de la violence présents dans notre système d'appropriation des biens. Ils contribuent très certainement à légitimer le recours, par les parents, à des conduites coercitives à l'endroit de leurs enfants et à renforcer la notion de "parent propriétaire " (Korbin, 1980 dans : Bouchard, 1981). 
Le "chacun pour soi" qui résulte inévitablement de ce type de contexte s'avère peu propice au développement communautaire et à l'apprentissage de conduites coopératives entre parents, amis, voisins ou collègues de travail. L'isolement des familles dans les secteurs à risque élevé et le manque d'appui qu'elles disent ressentir de la part de leur réseau social et de leur voisinage sont des phénomènes plus susceptibles d'apparaître dans les milieux qui partagent un tel système de valeurs (Bronfenbrenner, 1979).

La négligence serait également en partie une conséquence des systèmes économique, politique et culturel fondés sur une telle idéologie. Une illustration des plus frappantes en est le cas d'enfants avec "la clef autour du cou " (Latchkey children), ces enfants âgés de 8 à 12 ans laissés sans supervision après l'école ou ceux de 5 ans laissés avec leur frère ou leur souur à peine plus âgés qu'eux, et ce, jusqu'à ce que leurs parents reviennent de leur travail. Long et Long (1983, dans : Garbarino, 1983) stipulent que $30 \%$ des plaintes reçues dans les services sociaux concernaient des enfants manquant de supervision appropriée.

Des impératifs directement ou indirectement économiques nécessitent très souvent que les parents aient à prendre des décisions qui augmentent les risques dans l'environnement de l'enfant; de plus, les demandes d'adaptation sont alors exagérément élevées pour le niveau de développement du jeune. On sait déjà que les familles dont une femme est le soutien financier sont largement représentées dans la population des familles signalées pour mauvais traitements. Celles dont les deux parents travaillent et qui ont de jeunes enfants constituent un second groupe à risque. En fait, le travail des deux parents permet d'améliorer leur situation financière et d'échapper ainsi à la pauvreté ; en effet, $6.7 \%$ des familles où les deux parents travaillent vivent sous le seuil de paurreté, par rapport à $20.1 \%$ de celles où il n'y a qu'un seul pourvoyeur (Conseil national du Bien-être social, 1985). Il permet aussi, chez les plus nantis, d'augmenter leur quantité de biens, de consolider leurs acquis et de rechercher du prestige et du pouvoir dans la société.

\section{Là où les riches comme les pauvres sont tous deux perdants}

La recherche d'un meilleur statut, motivation suprême des idéologues conservateurs, encourage systématiquement le développement de schèmes de valeurs matérialistes, où l'accumulation des biens constitue bien souvent une fin en soi. À partir d'un sondage d'opinion, Burris 
(1979, dans : Garbarino, 1982) a constaté qu'avant 1975, les gens considéraient que les enfants étaient un élément plus important qu'une voiture pour promouvoir la qualité de la vie. En 1979, elle constata l'inverse. Ainsi, l'influence pernicieuse de nos systèmes économiques n'a pas que des effets délétères sur les pauvres, mais engendre également des contextes de survie, pour l'ensemble de la population, où l'impératif de l'accroissement du profit constitue une menace directe au développement d'environnements sûrs et stimulants pour les enfants. Au même titre qu'un niveau de pollution trop élevé indique un degré de détérioration de l'écosystème et appelle une transformation des pratiques technologiques des humains, un taux d'incidence très élevé de mauvais traitements envers les enfants représente un indice sérieux du niveau de dégradation de l'environnement physique, psychosocial et économique dans lequel les familles ont à s'adapter (Chamberland et Beaudry, sous presse).

Une réorientation profonde de nos valeurs et de nos systèmes politiques et économiques s'impose, afin de créer pour tous un environnement où l'activité et les ressources humaines seront considérées comme des éléments essentiels de la richesse d'une nation (Garbarino, 1984). Certains économistes prônent le développement de stratégies économiques variées à la fois pour stimuler l'expansion économique et provoquer une baisse significative du taux de pauvreté (voir à ce sujet les propositions de Garfinkel et Haveman, 1986). Ces mesures doivent toutefois assurer un environnement économique décent pour les enfants et exigent de recourir à des stratégies à long terme.

L'identification ainsi que la pertinence des systèmes de soutien que la société peut et doit offrir aux familles, par le biais d'une planification éclairée de politiques sociales cohérentes, 8 l'implantation de stratégies économiques plus préoccupées par la qualité de la vie que de l'accroissement du capital ainsi que la promotion du développement communautaire et social, relèvent d'une analyse holistique des systèmes sociaux gravitant autour de la relation parent-enfant. Le modèle écologique nous amène à préciser la nature complexe des interrelations entre, d'une part, les déterminants certes plus éloignés des milieux de vie immédiats de la famille et, d'autre part, les capacités personnelles de soutien du parent à promouvoir le développement optimal de l'enfant.

En somme, s'il est vrai que, pour que l'enfant développe une confiance personnelle et un sentiment de compétence, il doive être entouré d'adultes qui l'acceptent inconditionnellement, il est également vrai que l'État doit inconditionnellement assurer une sécurité matérielle et sociale aux parents pour qu'ils puissent l'aider à grandir (Cochran, 1985). 
Éduquer un enfant est coûteux en temps et exige de l'argent. C'est à se demander si nos sociétés peuvent toujours se permettre de se payer le luxe de l'enfance et, en corollaire, la paix et le bonheur des familles. Il y aura certainement des choix à faire et des priorités à identifier.

\section{Notes}

1 Ces taux, pour les États-Unis comme pour Montréal, excluent les cas d'abus sexuel.

2 Un voisinage correspond à un découpement administratif que l'on nomme secteur de recensement. Chaque secteur de recensement inclut en moyenne 740,24 enfants et 602,58 familles.

3 Les indicateurs macro-socio-économiques sont: le pourcentage de familles sous le seuil de pauvreté, le pourcentage de familles vivant au-dessus d'un seuil d'aisance, le pourcentage de familles monoparentales, le pourcentage de familles dont une femme est soutien financier, le pourcentage de familles résidant depuis moins d'un an dans leur logement, et le pourcentage de logements nécessitant des réparations majeures.

4 L'ensemble des résultats présentés dans cette section met en évidence des différences significatives entre les deux voisinages avec un $p<.05$. Pour plus de détails concernant la méthodologie ou les résultats, voir Chamberland, Bouchard et Beaudry (1986).

5 La violence et la négligence envers les enfants étant plus susceptibles de se produire en zones urbaines, l'évaluation comparative entre les États-Unis et le Canada sous-estime certainement l'écart entre les deux pays, puisque les taux américains représentent toutes les régions, tant urbaines que rurales.

6 Comparativement à 1980, il y aurait en $198420 \%$ de plus de familles à faible revenu. En 1984, près de $15 \%$ des familles et plus de $20 \%$ des enfants vivaient sous le seuil de pauvreté (Conseil national du Bien-être social, 1985).

7 En 1979, $20 \%$ des familles les plus pauvres (dernier quintile) possédaient $5.2 \%$ des ressources nationales (après les transferts gouvernementaux); en 1984, elles n'en possèdent plus que $4.2 \%$, ce qui constitue une diminution de $20 \%$ de leur revenu (Garfinkel et Haveman, 1986).

8 Voir, au Québec, le Rapport du Comité de consultation sur la politique familiale, en 1986, ou l'Avis sur la prévention en post-natal, produit en 1987 pour le Ministère de la santé et des services sociaux. Les différentes recommandations visent des cibles et suggèrent des stratégies diversifiées appelant une concertation entre ministères et institutions ; elles ont trait à une vaste gamme de services et de législations, tant dans le domaine de la santé et des services sociaux que ceux de la fiscalité, de l'habitation et du monde du travail. 


\section{Références bibliographiques}

American Humane Association, Highlights of Official Child Neglect and Abuse Reporting 1983, Denver, American Humane Association, 1985.

BELSKY, J., "Child maltreatment : an ecological integration ", American Psychologist, vol. $35, n^{\circ} 4,1980: 320-335$.

BOUCHARD, C., "Perspectives écologiques de la relation parent(s)-enfant: des compétences parentales aux compétences environnementales ", Apprentissage et socialisation, vol. 4, $\mathrm{n}^{\circ}$ 1, 1981: 4-23.

Bouchard, C., C. Chamberland et J. Beaudry, "Les mauvais traitements envers les enfants: une étude des facteurs macro et micro-économiques", dans: Jérôme Guar (éd.), Manuel québécois de psychologie communautaire, Chicoutimi, Gaëtan Morin, 1987 : 243-270.

Bronfenbrenner, U., The Ecology of Human Development, Cambridge (Mass.), Harvard University Press, 1979.

Chamberland, C. et J. Beaudry, Le paradigme écologique et l'étude des mauvais traitements envers les enfants, Montréal, Hurtubise H.M.H., (sous presse).

Chamberland, C., C. Bouchard et J. Beaudry, "Conduites abusives et négligentes envers les enfants : réalités canadienne et américaine", Revue canadienne des sciences du comportement, vol. 18, no 4, 1986 : 391-412.

Cochran, M., "Compétence personnelle chez les enfants et contextes ", communication présentée dans le cadre d'une série de séminaires sur la compétence, Laboratoire de recherche en écologie humaine et sociale, Université du Québec à Montréal, novembre 1985.

COCHRAN, M. et J.A. BRASSARD, "Child development and personal social networks", Child Development, vol. 50, 1979: 601-616.

Conseil national du Bien-être social, Profil de la pauvreté 1985, Ottawa, Gouvernement du Canada, 1985.

Egbuonu, B.S. et B. Starfield, "Child health and social status", Pediatrics, vol. 69, no 5, 1982: 550-557.

GARBARINO, J., "Child welfare and the economic crisis ", Child Welfare League of America, vol. 63, n० 1, $1984:$ 3-15.

GarbarIno, J., Children and Families in the Social Environment, New York, Aldine, 1982.

Garbarino, J., "Child maltreatment as a criminal justice problem », présentation au Congrès sur " Family Violence as a Criminal Justice Problem », Washington, octobre 1984.

Garbarino, J., Can American Families Afford the Luxury of Childhood?, document inédit, Pennsylvania State, Department of Individual and Family Studies, 1983.

Garbarino, J. et A. Crouter, "Defining the community context for parent-child relations: The correlates of child maltreatment ", Child Development, vol. 49, 1978 : 604-616.

Garbarino, J. et D. Sherman, "High-risk neighborhoods and high-risk families: The human ecology of child maltreatment ", Child Development, vol. 51, 1980 : 188-198. 
Garfinkel, I. et R. HAVEMAN, "Les politiques américaines contre la pauvreté : quelques propositions ", Revue internationale d'action communautaire, 16/ 56, 1986 : 77-93.

GIL, D.G., Violence against Children : Physical Child Abuse in the United States, Cambridge (Mass.), Harvard University Press, 1970.

MARTIN, G.M. et C. Messier, L'enfance maltraitée... ça existe aussi au Québec, Comité de la protection de la jeunesse, Ministère de la justice, Gouvernement du Québec, 1981.

Murray, C., Loosing Ground: American Social Policy, 1950-1980, New York, Basic Books, 1984.

Nersesian, W.S., M.R. Petit, R. Shaper, D. Lemieux et E. NAOR, "Childhood death and poverty: A study of all childhood deaths in Maine, 1976 to 1980 », Pediatrics, vol. 75, no 1, $1985:$ 41-50.

PARÉ, J., "Le Canada à l'américaine ", L'Actualité, vol. 11, no 6, juin 1986, p. 10.

Pelton, L.H., "Child abuse and neglect: The myth of classlessness", American Journal of Orthopsychiatry, vol. 48, no 4, 1978: 608-617.

Polansky, N.A, A.M. Chalmers, E. Bullenwieser et D. Williams, "The isolation of neglectful family ", American Journal of Orthopsychiatry, vol. 49, no 1, 1979 : 149-152.

Rocheleau, L., L. SéGuin, M. Cournoyer et C. Chamberland, Vivre avec un nourrisson : Avis et recommandations sur la prévention en post-natal, Comité FamilleEnfance de la Division Santé Communautaire de l'Association des hôpitaux du Québec, 1987, 460p. et 43p.

Rohner, B. et C. Nielsen, Parental Acceptance and Rejection : A Review and Annotated Bibliography of Research and Theory, New Haven (Conn.), Human Relations Area Files Press, 1978.

Strauss, M.A., R.J. Gelles et S.K. Steinmetz, Behind Closed Doors : Violence in the American Family, New York, Anchor Books, 1980. 